DPNU-02-08

hep-th/0204113

\title{
Localized gravity on de Sitter brane in five dimensions
}

\author{
Masato ITO * \\ Department of Physics, Nagoya University, Nagoya, JAPAN 464-8602
}

\begin{abstract}
We consider a single brane embedded in five dimensions with zero and positive cosmological constant. In this setup, the existence of $d S_{4}$ brane is allowed. We explore the gravitational fluctuations on the brane, it is pointed out that the usual four-dimensional gravity can be reproduced by a normalizable zero mode and that the continuous massive modes are separated by a mass gap from zero mode. We derive the relation among the cosmological constant in the brane, the five dimensional fundamental scale and Planck scale. Finally we show that nonzero small observed cosmological constant cannot be obtained in this setup.
\end{abstract}

*E-mail address: mito@eken.phys.nagoya-u.ac.jp 
Recent observation has indicated that the cosmological constant $\Lambda_{4}$ is a sufficiently small positive value, $\Lambda_{4} \sim\left(10^{-3} \mathrm{eV}\right)^{4}[1]$. Consequently it is natural to assume that we live in a de Sitter universe. However it is difficult to explain the reason for the smallness of $\Lambda_{4}$, so called cosmological constant problem. By using the warped extra dimension scenario by Randall and Sundrum [2], new suggestion for the cosmological constant problem was proposed.

Based on the assumption that physics of four dimensions should be explained by higher dimensional theory, the warped braneworlds with an infinite extra dimension are remarkable scenarios in the field of particle physics. Ref. [2] has led the conclusion that the gravity can be localized on flat 3-brane embedded in $A d S_{5}$, the usual four-dimensional gravity can be reproduced at large distance scale. This is because a normalizable zero mode exists and the effective four-dimensional Planck scale is finite. Due to fine-tuning between the bulk cosmological constant and the brane tension, the four-dimensional cosmological constant is vanishing. Furthermore, the close relation between $A d S / C F T$ correspondence and the Randall-Sundrum model is explored in the last couple of years $[3,4,5]$. Moreover it is expected that the warped braneworlds provide new scenario in the framework of phenomenological model buildings and cosmologies. We made the extension of the five-dimensional Randall-Sundrum model to $(5+n)$-dimensions, and analyzed the localization of gravity and the small correction terms to four-dimensional Newton's law $[6,7]$. Thus it is important to investigate the behavior of gravity in the warped braneworlds $[8,9,10,11,12,13,14,15,16,17]$. For instance, Karch and Randall had explored the behavior of the gravity on $d S_{4}$ brane and $A d S_{4}$ brane embedded in $A d S_{5}$ background [15]. In the case of $d S_{4}$, a normalizable zero mode of gravity exists. In the case of $A d S_{4}$, zero mode don't exist and massive modes have discrete eigenvalues due to the box-like volcano potential in Schrödinger equation.

Thus an important feature of the warped braneworld is the localization of gravity on the brane. Moreover, it is interesting to study whether nonzero small cosmological constant $\Lambda_{4}$ in brane can be obtained or not.

In this letter, we consider the braneworlds with zero and positive bulk cosmological constant in contrast to Karch and Randall [15]. Solving the Einstein equation of this setup, it is shown that there only exists the solution of $d S_{4}$ brane. We examine the resultant volcano potential in order to study the fluctuations of linearized gravity on a single $d S_{4}$ brane. Furthermore, we investigate the value of the cosmological constant $\Lambda_{4}$ in $d S_{4}$ brane.

The action of the setup, a single 3 -brane with the brane tension $V$ is located at $y=0$, is given by

$$
S=\int d^{4} x d y \sqrt{-G}\left(\frac{1}{2 \kappa_{5}^{2}} \mathcal{R}-\Lambda\right)-\int d^{4} x \sqrt{-g} V,
$$

where $\Lambda$ is the cosmological constant in the bulk and $\kappa_{5}^{2}$ is the five dimensional gravi- 
tational constant. The ansatz for metric in five dimensions is given by

$$
d s^{2}=a^{2}(y) g_{\mu \nu} d x^{\mu} d x^{\nu}+d y^{2} \equiv G_{M N} d x^{M} d x^{N}
$$

where $a(y)$ is a warp factor, it is assumed that the fifth dimension $y$ satisfies the $Z_{2}$ symmetry. The four-dimensional slice has a de Sitter metric as follows,

$$
g_{\mu \nu} d x^{\mu} d x^{\nu}=-d x_{0}^{2}+e^{2 \sqrt{\Lambda} x_{0}}\left(d x_{1}^{2}+d x_{2}^{2}+d x_{3}^{2}\right) .
$$

Here we define $3 \bar{\Lambda} \equiv \Lambda_{4} / M_{\mathrm{pl}}^{2}$, where $\Lambda_{4}$ and $M_{\mathrm{pl}}$ are four-dimensional cosmological constant and Planck scale, respectively. Moreover, Anti-de Sitter slices can be obtained by the following transformations: $x_{0} \rightarrow i x_{3}, x_{3} \rightarrow i x_{0}$, and $\sqrt{\bar{\Lambda}} \rightarrow i \sqrt{\bar{\Lambda}}$. Einstein equations of this setup are explicitly expressed as

$$
\left(\frac{a^{\prime}}{a}\right)^{2}-\frac{\bar{\Lambda}}{a^{2}}=-\frac{\kappa_{5}^{2}}{6} \Lambda
$$

and

$$
\frac{a^{\prime \prime}}{a}=-\frac{\kappa_{5}^{2}}{6} \Lambda-\frac{\kappa_{5}^{2}}{3} V \delta(y)
$$

Using the above equations, we can obtain the warp factor for $\Lambda<0, \Lambda=0$, and $\Lambda>0$. The solutions to the equations were first investigated in Ref. [8] which investigated the Einstein equations for a single brane or two branes embedded in the curved background.

For $\Lambda<0$, the solutions to $d S(\bar{\Lambda}>0)$ brane and $A d S(\bar{\Lambda}<0)$ brane have been analyzed by Karch and Randall [15]. Moreover, the case of $\bar{\Lambda}=0$ corresponds to the original Randall-Sundrum model [2]. For this reason, in this letter, we don't discuss the localization of gravity in the braneworlds with negative bulk cosmological constant.

For $\Lambda=0$, the solution of $\operatorname{AdS}(\bar{\Lambda}<0)$ brane cannot exist. Although the solution of $\bar{\Lambda}=0$ is allowed, the warp factor becomes a constant. Namely, the case is not interesting because of non-warped metric. However, the solution of $d S(\bar{\Lambda}>0)$ brane exists. The warp factor is given by

$$
a(y)=\sqrt{\bar{\Lambda}}(c-|y|),
$$

where $c$ is a positive integration constant. From Eq.(5), the jump condition at $y=0$ leads to the brane tension

$$
V=\frac{6}{\kappa_{5}^{2} c} .
$$

The form of warp factor in Eq.(6) yields the system of positive tension brane. 
For $\Lambda>0$, the solution of $d S(\bar{\Lambda}>0)$ brane is allowed. The warp factor can be expressed as [8]

$$
a(y)=L \sqrt{\bar{\Lambda}} \sin \frac{c-|y|}{L}, \quad L=\sqrt{\frac{6}{k_{5}^{2} \Lambda}} .
$$

From Eq.(4), note that the solutions of $\bar{\Lambda}=0$ and $\bar{\Lambda}<0$ cannot be allowed. Furthermore, the brane tension takes the form as follows

$$
V=\frac{6}{\kappa_{5}^{2} L} \cot \frac{c}{L}
$$

Thus the positive tension brane can be obtained by taking positive constant $c$. From Eqs.(6) and (8), it implies that $c$ is the distance between the brane and the horizon. Moreover, the normalization condition of $a(0)=1$ leads to the relation between $\bar{\Lambda}$ and $c$. Consequently, we have $\bar{\Lambda}=c^{-2}$ for $\Lambda=0$, and $\bar{\Lambda}=L^{-2} \sin ^{-2} c / L$ for $\Lambda>0$. From Eqs.(7) and (9), we have $\bar{\Lambda}=\left(\kappa_{5}^{2} V / 6\right)^{2}$ for $\Lambda=0$ and $\bar{\Lambda}=\left(\kappa_{5}^{2} V / 6\right)^{2}+L^{-2}$ for $\Lambda>0$. Taking the limit of large $L$ (small $\Lambda$ ), the warp factor of Eq.(8) approaches the one of Eq.(6). For the limit of $c / L \ll 1$, Eq.(9) is consistent with Eq.(7).

As mentioned above, in setup considered here, it turns out that a $d S_{4}$ brane can be allowed in five dimensions with zero and positive cosmological constant. Below, in order to study the behavior of gravity we examine the potential in wave equation for gravitational fluctuation.

Taking $g_{\mu \nu}+a^{-2}(y) h_{\mu \nu}(x, y)$ as the gravitational fluctuation around the background metric, we can obtain the wave equation for gravity. By imposing transverse-traceless gauge conditions for these fluctuations, a separation such as $h_{\mu \nu}(x, y)=h_{\mu \nu}(x) \psi(y)$ yields the wave function for gravity as follows

$$
\frac{d^{2}}{d y^{2}} \psi+\left[-2 \frac{a^{\prime \prime}}{a}-2\left(\frac{a^{\prime}}{a}\right)^{2}+m^{2} a^{-2}\right] \psi=0 .
$$

Here we used wave equation of the four-dimensional gravity in curved background, ( $\square+$ $\left.2 \Lambda_{4} / M_{\mathrm{pl}}^{2}\right) h_{\mu \nu}=m^{2} h_{\mu \nu}$, where $\square$ is the four-dimensional covariant d'Alembertian and $\mathrm{m}^{2}$ corresponds to the mass of four-dimensional gravity. Transforming to conformally flat coordinate $z=\int d y a^{-1}(y)$ and performing replacement of $\psi(y)=a^{1 / 2}(y) \tilde{\psi}(z)$, we have the familiar Schrödinger equation. For the cases of $\Lambda=0$ and $\Lambda>0$, we show that the relation between $y$-coordinate and $z$-coordinate and the resultant volcano potential in the Schrödinger equation. The conformally $z$ coordinate is given by

$$
\begin{aligned}
& \Lambda=0: z(y)=\operatorname{sgn}(y) \frac{1}{\sqrt{\bar{\Lambda}}}\left\{\log \left(\frac{1}{c-|y|}\right)-z_{0} \sqrt{\bar{\Lambda}}\right\} \\
& \Lambda>0: z(y)=\operatorname{sgn}(y) \frac{1}{\sqrt{\bar{\Lambda}}}\left\{\operatorname{arccosh}\left(\frac{1}{\sin \frac{c-|y|}{L}}\right)-z_{0} \sqrt{\bar{\Lambda}}\right\},
\end{aligned}
$$




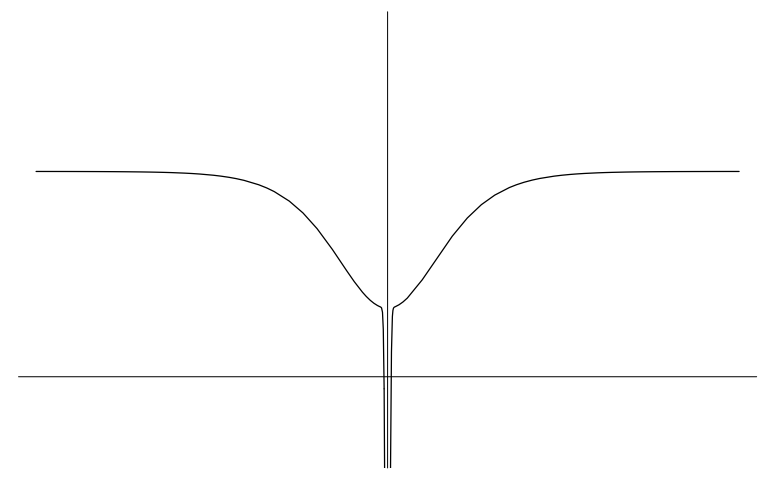

Figure 1: Volcano potential in $d S$ brane for $\Lambda>0$

where the constant $z_{0}$ is given by

$$
\begin{aligned}
& \Lambda=0 \quad: \quad z_{0}=\frac{1}{\sqrt{\bar{\Lambda}}} \log \frac{1}{c} \\
& \Lambda>0: z_{0}=\frac{1}{\sqrt{\bar{\Lambda}}} \operatorname{arccosh}\left(\frac{1}{\sin \frac{c}{L}}\right) .
\end{aligned}
$$

Thus the $z$ coordinate runs from $-\infty$ to $+\infty$. The warp factors can be rewritten in terms of $z$, accordingly, $a(z)=e^{-\sqrt{\Lambda}|z|}$ for $\Lambda=0$ and $a(z)=L \sqrt{\bar{\Lambda}} / \cosh \left(\sqrt{\bar{\Lambda}}\left(z_{0}+|z|\right)\right)$ for $\Lambda>0$. Using Eqs.(6) and (8), Eq.(10) is expressed in terms of $z$

$$
\left[-\frac{d^{2}}{d z^{2}}+V(z)\right] \tilde{\psi}=m^{2} \tilde{\psi},
$$

where the volcano potential for $\Lambda=0$ and $\Lambda>0$ is given by

$$
\begin{aligned}
& \Lambda=0: V(z)=\frac{9}{4} \bar{\Lambda}-3 \sqrt{\bar{\Lambda}} \delta(z) \\
& \Lambda>0: V(z)=\frac{9}{4} \bar{\Lambda}-\frac{15}{4} \frac{\bar{\Lambda}}{\cosh ^{2} \sqrt{\bar{\Lambda}}\left(z_{0}+|z|\right)}-3 \sqrt{\bar{\Lambda}} \tanh z_{0} \sqrt{\bar{\Lambda}} \delta(z) .
\end{aligned}
$$

In the case of $\Lambda=0$ the volcano potential is a positive constant. In the case of $\Lambda>0$ as shown in Fig.1, the potential approaches a non-zero constant $9 \bar{\Lambda} / 4$ for $|z| \rightarrow \infty$. Note that there is no blow-up at the horizon in contrast to setup of $A d S_{4}$ brane embedded in $A d S_{5}$ analyzed by Karch and Randall [15]. Since the Hamiltonian in Eq.(15) is a positive definite value, the eigenvalue $m^{2} \geq 0$ [11].

For $\Lambda=0$, solving the Schrödinger equation imposed by the jump condition at $z=$ 0 , the zero mode wave function is given by $\tilde{\psi}_{0}(z)=(3 \sqrt{\bar{\Lambda}} / 2)^{1 / 2} e^{-\frac{3}{2} \sqrt{\Lambda}|z|}$. Since there is a normalizable zero mode wave function, the localization of gravity occurs on the brane. Namely, the effective four-dimensional Planck scale is finite value. The massive mode 
wave functions for $0<m^{2}<9 \bar{\Lambda} / 4$ are given by $\tilde{\psi}_{m} \sim \exp \left[-\sqrt{9 \bar{\Lambda} / 4-m^{2}}|z|\right]$, however, these modes do not exist there. This is because these modes aren't consistent with jump condition. For $m^{2} \geq 9 \bar{\Lambda} / 4$, the wave functions become plane waves with continuous modes. Thus a zero mode mode is separated by a mass gap $9 \bar{\Lambda} / 4$ from the continuous massive modes. The gravitational potential between two unit masses via zero mode is given by $V(r) \sim \kappa_{5}^{2}\left|\tilde{\psi}_{0}(0)\right|^{2} / r=3 \kappa_{5}^{2} \sqrt{\bar{\Lambda}} / 2 r$. The contributions of massive modes are described in summary. Therefore we can obtain the effective four-dimensional Planck scale $M_{\mathrm{pl}}^{-2}=3 \kappa_{5}^{2} \sqrt{\bar{\Lambda}} / 2$. Consequently, the four-dimensional cosmological constant $\Lambda_{4}$ is given by

$$
\Lambda_{4}=\frac{4}{9} M_{\mathrm{pl}}^{4}\left(\frac{M}{M_{\mathrm{pl}}}\right)^{6} .
$$

Here we used $\bar{\Lambda} \sim \Lambda_{4} / M_{\mathrm{pl}}^{2}$ and $\kappa_{5}^{2} \equiv M^{-3}$, where $M$ is the fundamental scale of five dimensions. Note that $\Lambda_{4}$ is in proportion to sixth powers of ratio $M / M_{\mathrm{pl}}$, Eq.(18) is consistent with $\Lambda_{4}$ in warped compactification of Type IIB string theory [18]. The observed value of $\Lambda_{4}$ leads to the ratio $M / M_{\mathrm{pl}} \sim 10^{-20}$, namely, we obtain $M=$ $\mathcal{O}(100 \mathrm{MeV})$. Thus this case is phenomenologically ruled out. Setting $M=\mathcal{O}(\mathrm{TeV})$, the cosmological constant in brane cannot be consistent with the observed value.

For $\Lambda>0$, the general solution of the Schrödinger equation with potential of Eq.(17) is expressed as

$$
\begin{aligned}
& \tilde{\psi}_{m}(z)=A\left[\cosh \sqrt{\bar{\Lambda}}\left(z_{0}+|z|\right)\right]_{2}^{-\rho} F_{1}\left(\rho-\frac{3}{2}, \rho+\frac{5}{2} ; 1+\rho ; \frac{1-\tanh \sqrt{\bar{\Lambda}}\left(z_{0}+|z|\right)}{2}\right) \\
& +B\left[\cosh \sqrt{\bar{\Lambda}}\left(z_{0}+|z|\right)\right]_{2}^{\rho} F_{1}\left(-\rho-\frac{3}{2},-\rho+\frac{5}{2} ; 1-\rho ; \frac{1-\tanh \sqrt{\bar{\Lambda}}\left(z_{0}+|z|\right)}{2}\right)
\end{aligned}
$$

where $\rho=\sqrt{9 / 4-m^{2} / \bar{\Lambda}}$ and $A, B$ are the normalization factors. Taking account into the divergence at $|z| \rightarrow \infty$, both $B=0$ and $3 / 2-\rho=n$ can be required by the finiteness of hypergeometric function, where $n \in \mathbf{Z}_{0}^{+}$. Namely, the cases of $n=0,1$ are allowed. For $n=0\left(\rho=3 / 2, m^{2}=0\right)$, this corresponds to a zero mode of gravity and the corresponding wave function is given by $\tilde{\psi}_{0}(z) \sim \cosh ^{-3 / 2} \sqrt{\bar{\Lambda}}\left(z_{0}+|z|\right)$, which is consistent with the jump condition at $z=0$. For $n=1\left(\rho=1 / 2, m^{2}=2 \bar{\Lambda}\right)$, the massive modes take the following form, $\tilde{\psi}_{m} \sim \sinh \sqrt{\bar{\Lambda}}\left(z_{0}+|z|\right) \cosh ^{-3 / 2} \sqrt{\bar{\Lambda}}\left(z_{0}+|z|\right)$, however, the wave function isn't consistent with the jump condition. Namely, in the region of $0 \leq m^{2}<9 \bar{\Lambda} / 4$, there is a normalizable zero mode. For $m^{2}>9 \bar{\Lambda} / 4$, since $\rho$ is imaginary number, the solutions of Eq.(19) are plane waves with continuous modes at large $z$. Also in this case there exists a mass gap between the zero mode and massive modes, the onset of massive mode starts from $\sqrt{9 \bar{\Lambda} / 4}$. The behavior of Newton potential via massive modes is described in summary. At $r \gg r_{0}=2 / 3 \sqrt{\bar{\Lambda}}$, a 
zero mode generates the usual Newton's law. Estimating the normalization factor of zero mode wave function, we can obtain the cosmological constant $\Lambda_{4}$ in $d S$ brane as follows,

$$
\Lambda_{4}=M_{\mathrm{pl}}^{4}\left(\frac{M}{M_{\mathrm{pl}}}\right)^{6}\left(1+\epsilon^{2}\right)^{3}\left(\frac{\pi}{2}-\frac{\epsilon}{1+\epsilon^{2}}-\arctan \epsilon\right)^{2}, \quad \epsilon \equiv \frac{L V}{6 M^{3}} .
$$

Note that the value of $\Lambda_{4}$ is determined by the bulk curvature $L$ when $M$ and $V$ are fixed. For instance, the limit of $\epsilon \rightarrow \infty$ leads to

$$
\Lambda_{4}=\frac{4}{9} M_{\mathrm{pl}}^{4}\left(\frac{M}{M_{\mathrm{pl}}}\right)^{6}
$$

where this situation corresponds to the case of $\Lambda=0$. Moreover the case of $\epsilon=0(\Lambda \rightarrow$ $\infty)$ leads to

$$
\Lambda_{4}=\frac{\pi^{2}}{4} M_{\mathrm{pl}}^{4}\left(\frac{M}{M_{\mathrm{pl}}}\right)^{6}
$$

Thus, for arbitrary value of the cosmological constant in the bulk, we obtain approximately $\Lambda_{4} \sim \mathcal{O}(1) \times M_{\mathrm{pl}}^{4}\left(M / M_{\mathrm{pl}}\right)^{6}$. Similar to the case of $\Lambda=0$, we must set $M=\mathcal{O}(100 \mathrm{MeV})$ in order to obtain the observed value of $\Lambda_{4}$.

In summary, we considered a single positive tension 3-brane embedded in fivedimensional world with zero $(\Lambda=0)$ and positive $(\Lambda>0)$ cosmological constant. Solving Einstein equations of the setup, $d S$ brane $(\bar{\Lambda}>0)$ is allowed, and the cases of flat brane $(\bar{\Lambda}=0)$ and $A d S$ brane $(\bar{\Lambda}<0)$ are ruled out. Furthermore, we investigated whether nonzero small cosmological constant in brane can be obtained or not.

In the case of $d S$ brane in $\Lambda=0$, the resultant volcano potential is a positive nonzero constant, and positive tension brane gives rise to a delta function of attractive force. Furthermore, there is a mass gap between zero mode and continuous massive mode. We derived the relation between the cosmological constant in the brane and the five-dimensional fundamental scale. In order to obtain nonzero small observed cosmological constant, it turns out that the five-dimensional fundamental scale must be the order of a few MeV scale.

In the case of $d S$ brane in $\Lambda>0$, the resultant volcano potential approaches a positive non-zero constant at large conformally coordinate $z$. In similar to the above case, there exists a mass gap between zero mode and massive modes. It is shown that the cosmological constant in brane depends on the value of bulk curvature. Similar to the case of $\Lambda=0$, nonzero small observed cosmological constant is tuned by the five dimensional scale of a few $\mathrm{MeV}$ scale.

We must describe some comments with respect to the behaviors of Newton potential via the massive modes. For $\Lambda=0$ and $\Lambda>0$, taking into account of the massive modes, 
the Newton potential on the $d S$ brane were investigated in Ref.[19]. The continuous massive modes separated by the mass gap from zero mode give the correction terms to the Newton potential. At the distance larger than the inverse of lowest massive mode and smaller than Hubble scale $H=\sqrt{\bar{\Lambda}}$, the five dimensional gravity appears on the brane due to dominance of correction terms.

Thus the localization of gravity on the brane occurs in the setup considered here, however, nonzero small observed cosmological constant cannot be obtained. The setup of $d S$ brane embedded in $\Lambda=0$ and $\Lambda>0$ can be ruled out from the viewpoint of astrophysics measurements. Here we give some comments. Although de Sitter bulk may be unusual in the cosmological context, there are a number of investigations of $d S / C F T$ correspondence inspired by $A d S / C F T$. There are many works exploring fully satisfactory $d S$ solution of string theory, it is expected that $d S$ may provide several solutions to the cosmological constant problem or quantum gravity.

\section{References}

[1] S. Perlmutter et al. [Supernova Cosmology Project Collaboration], "Measurements of Omega and Lambda from 42 High-Redshift Supernovae," Astrophys. J. 517, 565 (1999) [astro-ph/9812133].

[2] L. Randall and R. Sundrum, "An alternative to compactification," Phys. Rev. Lett. 83, 4690 (1999) [hep-th/9906064].

[3] J. Maldacena, "The large $N$ limit of superconformal field theories and supergravity," Adv. Theor. Math. Phys. 2, 231 (1998) [hep-th/9711200].

[4] E. Witten, "Anti-de Sitter space and holography," Adv. Theor. Math. Phys. 2, 253 (1998) [hep-th/9802150].

[5] S. S. Gubser, I. R. Klebanov and A. M. Polyakov, "Gauge theory correlators from non-critical string theory," Phys. Lett. B 428, 105 (1998) [hep-th/9802109].

[6] M. Ito, "Newton's law in braneworlds with an infinite extra dimension," Phys. Lett. B 528, 269 (2002) [hep-th/0112224].

[7] M. Ito, "Warped geometry in higher dimensions with an orbifold extra dimension," Phys. Rev. D 64, 124021 (2001) [hep-th/0105186].

[8] N. Kaloper, "Bent domain walls as braneworlds," Phys. Rev. D 60, 123506 (1999) [arXiv:hep-th/9905210].

[9] J. Lykken and L. Randall, "The shape of gravity," JHEP 0006, 014 (2000) [hepth/9908076]. 
[10] S. B. Giddings, E. Katz and L. Randall, "Linearized gravity in brane backgrounds," JHEP 0003, 023 (2000) [hep-th/0002091].

[11] C. Csaki, J. Erlich, T. J. Hollowood and Y. Shirman, "Universal aspects of gravity localized on thick branes," Nucl. Phys. B 581, 309 (2000) [hep-th/0001033].

[12] M. D. Schwartz, "The emergence of localized gravity," Phys. Lett. B 502, 223 (2001) [hep-th/0011177].

[13] M. Gremm, "Thick domain walls and singular spaces," Phys. Rev. D 62, 044017 (2000) [hep-th/0002040].

[14] M. Ito, "Linearized gravity in flat braneworlds with anisotropic brane tension," [hep-th/0202166].

[15] A. Karch and L. Randall, "Locally localized gravity," JHEP 0105, 008 (2001) [hep-th/0011156].

[16] A. Miemiec, "A power law for the lowest eigenvalue in localized massive gravity," Fortsch. Phys. 49, 747 (2001) [hep-th/0011160].

[17] I. Brevik, K. Ghoroku, S. D. Odintsov and M. Yahiro, "Localization of gravity on brane embedded in $\operatorname{AdS}(5)$ and dS(5)," [hep-th/0204066].

[18] P. Berglund, T. Hubsch and D. Minic, "Relating the cosmological constant and supersymmetry breaking in warped compactifications of IIB string theory," [hepth/0201187].

[19] A. Kehagias and K. Tamvakis, "Graviton localization and Newton law for a dS(4) brane in 5D bulk," Class. Quant. Grav. 19, L185 (2002) [arXiv:hep-th/0205009]. 